\title{
Action plans can interact to hinder or facilitate reach performance
}

\author{
Lisa R. Fournier ${ }^{1} \cdot$ Matthew D. Wiediger $^{2} \cdot$ Ezana F. Taddese $^{1}$
}

Published online: 11 July 2015

(C) The Psychonomic Society, Inc. 2015

\begin{abstract}
Executing a reach action can be delayed while retaining another action in working memory (WM) if the two action plans partly overlap rather than do not overlap. This delay (partial repetition cost) occurs when reach responses are under cognitive control. In this study, we investigated whether facilitation (a partial repetition benefit) occurs when reach responses are automatic. We also examined whether the hemisphere controlling the limb or selection of the preferred limb (based on a free-reach task) influences reach performance when the actions partly overlap. Leftand right-handers reached to different stimulus locations to the left and right of body midline with their ipsilateral hand while maintaining an action plan in WM that required the same or the different hand. The results showed a partial repetition benefit for spatially compatible reaches to left and right stimulus locations far from the body midline, but not for those near the body midline. Also, no partial repetition cost was found at any of the stimulus-reach locations. This indicates that automatic reach responses that partly overlap with an action plan maintained in WM are not delayed, but instead can be facilitated (partial repetition benefit). The roles of hemisphere and reach-hand preference in action control and the importance of the degree of feature overlap in obtaining a partial repetition benefit (and cost) are discussed.
\end{abstract}

Electronic supplementary material The online version of this article (doi:10.3758/s13414-015-0959-5) contains supplementary material, which is available to authorized users.

Lisa R. Fournier

lfournier@wsu.edu

1 Washington State University, Pullman, WA, USA

2 MacMurray College, Jacksonville, IL, USA
Keywords Cognitive control · Automaticity · Goal-directed movements $\cdot$ Perception and action

Goal-directed actions, such as operating a computer and cooking a meal, require action planning. We must decide what to do and when to do it (Keele, 1968; Lashley, 1951; Miller, Galanter, \& Pribram, 1960). Sometimes we suspend the execution of one action plan to execute another. For example, one may plan to remove a pan of chicken close to burning from the stove top, but reaching to an object about to fall out of the cupboard onto one's head takes precedence. Wiediger and Fournier (2008) showed that executing a reach action can be delayed while retaining another action in working memory (WM) if the two action plans partly overlap (e.g., both actions require the left hand) versus do not overlap (e.g., the actions require different hands). These delays occurred when reach responses were under cognitive control (Passingham \& Toni, 2001). It is unclear, however, whether reach actions under automatic control (Logan, 1988) would be facilitated, instead of delayed. In the present study, we addressed this question. We also examined whether the hemisphere controlling the limb (Gonzalez, Ganel, \& Goodale, 2006) or selection of the preferred limb (Stins, Kadar, \& Costall, 2001) influences reach performance when the actions partly overlap. Understanding when action plans interact to hinder or facilitate reach responses will allow us to better predict performance and better understand how action plans are represented.

Actions directed toward a goal are defined as controlled (Logan, 1988; Logan \& Crump, 2011; Miller et al., 1960). For example, the act of typing by either skilled or unskilled typists is controlled, because the typist chooses to do it; it does not occur by accident (Logan \& Crump, 2011). Actions under cognitive control are accessed by a time-consuming process of applying a general algorithm or rule(s) held in WM (indirect 
route). In contrast, actions under automatic control are rapidly and directly accessed as stored solutions in long-term memory and can be specified directly and unconditionally by a stimulus (direct route; Barber \& O’Leary, 1997; de Jong, Liang, \& Lauber, 1994; Eimer, Hommel, \& Prinz, 1995; Greenwald, 2003; Greenwald \& Shulman, 1973; Kornblum, Hasbroucq, \& Osman, 1990; Newell \& Rosenbloom, 1981; Stürmer, Leuthold, Soetens, Schröter, \& Sommer, 2002; Tagliabue, Zorzi, Umiltà, \& Bassignani, 2000; Umiltà \& Zorzi, 1997). These processes are at opposite ends of the same processing continuum and exist as a matter of degree - not as all-or-none (Anderson, 1992; Cohen, Dunbar, \& McClelland, 1990; Logan, 1988; Macleod \& Dunbar, 1988; Newell \& Rosenbloom, 1981). Thus, some goal-directed actions are more automatic than others or require more cognitive demand than others.

Executing an action (a vocal response, keypress response, or reach response) can be delayed if it partly overlaps with an action plan maintained in WM, at least when the ongoing action is under cognitive control (Fournier, Behmer, \& Stubblefield, 2014a; Hommel, Müsseler, Aschersleben, \& Prinz, 2001; Meyer \& Gordon, 1985; Sevald \& Dell, 1994; Stoet \& Hommel, 1999; Yaniv, Meyer, Gordon, Huff, \& Sevald, 1990). For example, executing a left-hand keypress conditional on stimulus identity (a red target) is delayed if it shares a feature code ("left") with an action plan maintained in WM ("left hand move up"), as compared to when it does not ("right hand move up"; Mattson \& Fournier, 2008; Stoet \& Hommel, 1999). This delay is referred to as a partial repetition cost. These costs are assumed to occur when a feature code from the current action plan reactivates (primes) the action plan retained in WM. The action features are assumed to be integrated in the action plan, so reactivating (priming) one feature activates the other features with which it is integrated. This leads to temporary confusion (code confusion) as to which action plan is relevant for the current task: the current plan or the plan maintained in WM (Fournier \& Gallimore, 2013; Fournier, Gallimore, Feiszli, \& Logan, 2014b; Hommel, 2004, 2005; Mattson \& Fournier, 2008; Mattson, Fournier, \& Behmer, 2012). As a result, the irrelevant feature code or action plan must be inhibited, and the time required to inhibit it delays selection of the action plan for the current task (see also Sevald \& Dell, 1994).

Wiediger and Fournier (2008) suggested that partial repetition costs occur only for actions under cognitive control (see also Fournier et al., 2010; Thomaschke, Hopkins, \& Miall, 2012a, b), and hence code confusion is limited to actions that impose a demand on WM. If the current action is automatic, it should not reactivate other plans in WM with similar features. As a result, no code confusion should occur, and no partial repetition cost should be observed. However, the current, automatic action can benefit from priming by the action plan maintained in WM, if they overlap. These conclusions were based on reach actions directed to a spatially compatible stimulus that either was or was not conditional on stimulus identity. Wiediger and Fournier found partial repetition costs when left or right reach responses to a stimulus depended on the color of the stimulus [reach to the red stimulus (target), not the green stimulus] and on the location of the target stimulus relative to body midline (reach with the left hand if the target is on the left, and with the right hand if it is on the right). In contrast, partial repetition costs were not consistently found (in two experiments) when response selection depended only on stimulus location (reach with the left hand if the stimulus is on the left, and with the right hand if it is on the right). In this case, right-handed participants showed a small but significant partial repetition cost for left-hand reaches $17^{\circ}$ to the left of body midline, but showed either no cost or a partial repetition benefit for right-hand reaches $17^{\circ}$ to the right of body midline.

The finding of a cost or benefit contingent on the left or right spatial compatibility of the response is interesting, because the spatial position of the task-relevant stimulus should automatically activate the correct reach response (Fitts \& Seeger, 1953; Umiltà \& Nicoletti, 1990). That is, left or right hand reach responses should be specified directly by the spatial stimulus (left or right), without the involvement of WM (direct route; de Jong et al., 1994; Eimer, 1995; Eimer et al., 1995; Kornblum \& Lee, 1995; Proctor, Lu, Wang, \& Dutta, 1995; Simon, 1990; Tagliabue et al., 2000). Wiediger and Fournier (2008) concluded that spatially compatible reaches $17^{\circ}$ to the left of body midline were not executed automatically by right-handed participants in their study, but reaches $17^{\circ}$ to the right of body midline were. They offered two equally plausible accounts for their findings. First, they suggested that the degree of cognitive and automatic control may be influenced by the hemisphere controlling the reach. This account is consistent with evidence that the execution of reach and grasp actions controlled by the right hemisphere (left-hand actions) relies more on cognitive control mechanisms, whereas those controlled by the left hemisphere (right-hand actions) rely more on automatic mechanisms (Elliot \& Chua, 1996; Fisk \& Goodale, 1988; Gonzalez et al., 2006; Radoeva, Cohen, Corballis, Lukovits, \& Koleva, 2005; Todor \& Cisneros, 1985). Thus, spatially compatible reaches with the right hand, but not with the left hand, can be executed automatically, and hence should not lead to a partial repetition cost - but could lead to a benefit. Second, they suggested that reach-hand preference could account for their findings. This account assumes that spatially compatible reaches with the left hand require more cognitive control if righthanded individuals naturally reach with their right hand across body midline to this location. Inhibiting a natural reach response may be analogous to inhibiting automatically evoked saccades to abrupt onsets (e.g., Hallet, 1978; Theeuwes, Kramer, Hahn, \& Irwin, 1998). Thus, the reach-hand preference, not simply the spatial compatibility 
of the response, influences the degree of automatic and cognitive control, and hence whether a partial repetition benefit or cost will be observed.

Consistent with the reach-hand preference account, Stins et al. (2001) showed that left- and right-handed participants preferred to use their dominant hand to reach to a stimulus located less than $23^{\circ}$ to the left or the right of body midline. Also, they showed that regardless of handedness, reaches to a stimulus greater than $23^{\circ}$ to the left or to the right of body midline were almost exclusively executed with the spatially compatible hand (see also Gabbard \& Rabb, 2001; Helbig \& Gabbard, 2004). They and others suggested that responses are naturally used that minimize the curvature and joint amplitude of a reach (Kim, Buchanan, \& Gabbard, 2011; Kim, Gabbard, Ryu, \& Buchanan, 2007), which may maximize comfort in achieving the response goal (Rosenbaum, Chapman, Weigelt, Weiss, \& van der Wel, 2012). Wiediger and Fournier (2008) suggested that reach responses that are naturally used (due to hand dominance and/or comfort) may be more automatic than reach responses that are not.

\section{Study design and rationale}

This study used the partial repetition paradigm (Stoet \& Hommel, 1999) to examine whether partial repetition benefits occur for spatially compatible reach responses that are assumed to be automatic (e.g., Tagliabue et al., 2000; Umiltà \& Nicoletti, 1990). We also examined whether these benefits are influenced by the hemisphere controlling the reaching limb or by reach-hand preference (i.e., the hand one would naturally use). Two different visual events (A and B) were presented sequentially to left- and right-handed participants. When Event A was presented, participants planned and maintained keypress responses with their left or right hand, based on its identity. While they maintained the action plan to Event A in WM, Event B appeared, calling for an immediate, ipsilateral reach response to its detected location either $15^{\circ}, 35^{\circ}$, or $54^{\circ}$ to the left or right of the body midline. After participants executed a speeded reach to the Event B location, they executed the planned action for Event A. The main manipulation was whether the hand used to reach to Event $B$ was the same as the one required to respond to Event A (feature overlap) or not (no feature overlap). Because spatially compatible reach responses should be automatic, partial repetition benefits as opposed to costs were expected. If the hemisphere controlling the hand or reach-hand preference also influences the degree of automatic control, then partial repetition benefits should be contingent on the stimulus-reach location.

We examined whether the factor of Feature Overlap interacted with handedness (left or right dominant), reach hand (reach to Event $\mathrm{B}$ with the dominant or nondominant hand), and Event $\mathrm{B}$ eccentricity $\left(15^{\circ}, 35^{\circ}\right.$, or $\left.54^{\circ}\right)$. If the left hemisphere is specialized for automatic control and the right hemisphere is specialized for the cognitive control of action (Gonzalez et al., 2006), then a partial repetition benefit should occur for spatially compatible reaches to the right but not to the left of body midline, regardless of whether participants are left- or right-handed. Also, a partial repetition cost should occur for left-hand reaches, regardless of whether participants are left- or right-handed. In contrast, if reach-hand preference (based on the free-reach findings described earlier) influences whether reaches are under automatic or cognitive control, then a partial repetition benefit should occur for all spatially compatible reaches with the dominant and nondominant hands, except for reaches with the nondominant hand to the $15^{\circ}$ location. Only the latter reach response would not be preferred (naturally selected) and should require more cognitive control, resulting in a partial repetition cost. We also incorporated a noplanning condition to serve as a single-task baseline. Before engaging in this action-planning task, participants completed a free-reach task that confirmed that the hand that participants would naturally choose (i.e., preferred) to reach to the different Event B locations was consistent with the findings from previous research.

\section{Method}

\section{Participants}

Sixty undergraduates from Washington State University, solicited on the basis of subjective reports of left-hand dominance $(n=30)$ and right-hand dominance $(n=30)$, participated for optional extra credit in psychology classes. The sample size chosen was approximately twice the size used in previous studies using the same paradigm (see, e.g., Wiediger \& Fournier, 2008), to ensure sufficient power. All participants had at least 20/60 visual acuity as assessed by a Snellen chart, and 30 were right-handed and 30 were left-handed, as assessed by the Edinburgh Handedness Inventory (Oldfield, 1971) and the Lateral Preference Inventory (Coren, 1993). Six participants (five right-handed and one left-handed) in the action-planning task were excluded for failing to follow instructions.

\section{Apparatus}

A CRT was centered $75 \mathrm{~cm}$ in front of the participant. Six pedestals $(39 \mathrm{~cm}$ tall and $10 \mathrm{~cm}$ wide, covered with black construction paper) were arranged on a table top $50 \mathrm{~cm}$ from the participant's body midline, in an arc approximately $15^{\circ}$, $35^{\circ}$, and $54^{\circ}$ to the left and right of the participant's body midline (see Fig. 1). Each pedestal contained a circular button (ENV-123AM Pigeon Key, radius of $1.25 \mathrm{~cm}$, positioned $32 \mathrm{~cm}$ above the table top) that could be illuminated. The 


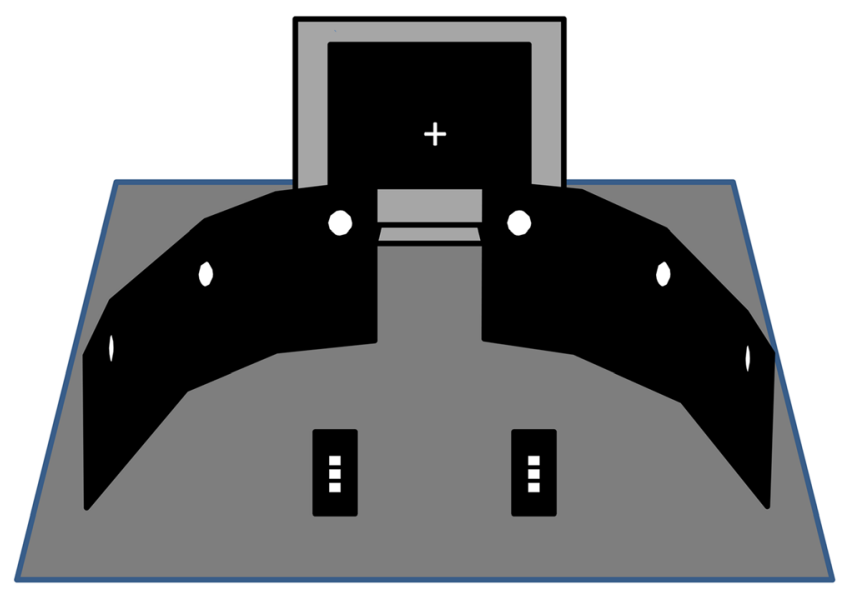

Fig. 1 Diagram of the apparatus used for the free-reach task and the action-planning task. All reach responses in the free-reach and actionplanning tasks (Event B) were made from the keypads (toward the bottom of the figure) to an illuminated, circular response button located on the black panels. The CRT displayed start instructions and the fixation cross in the free-reach and action-planning tasks; it also displayed Event $\mathrm{A}$ and performance feedback in the action-planning task

tension of each button was set between 12 and $15 \mathrm{~g}$ of pressure, with a maximum depression depth of $1 / 8 \mathrm{~cm}$. Two keypads were placed on the table top: one located to the left and the other to the right of the participant's body midline, $20 \mathrm{~cm}$ from the table edge. A distance of $22 \mathrm{~cm}$ separated the keypads. Each keypad had three keys $(1 \mathrm{~cm} \times 1 \mathrm{~cm}$ in size, separated by $0.2 \mathrm{~cm}$ ) oriented in a vertical array. The key in the middle of each keypad was referred to as the home key. The reaching distance from the home keys to each of the circular buttons was $19 \mathrm{~cm}$.

\section{Stimuli, responses, and procedure for the free-reach task}

To assess natural hand selections to the different light locations, the frequency with which participants used their dominant hand to reach to the illuminated light locations was recorded. Importantly, the choice to reach with the dominant hand to the different light locations was made freely by the participant. All stimuli were white on a black background. Each trial began with an initiation screen on the CRT that presented a central fixation cross $\left(1.0^{\circ}\right.$ of visual angle) and a message that read "Press the home keys to continue." Participants placed their left and right index fingers on the center keys of the respective left and right keypads, pressing both center keys simultaneously to initiate a trial. One second later, one of the six circular buttons on the pedestals illuminated. Participants freely chose a hand to reach to and depress the illuminated button as quickly as possible. Participants had $10 \mathrm{~s}$ to depress the button. After depressing the button, or after $10 \mathrm{~s}$ had elapsed, the trial ended. Then the initiation screen for the next trial appeared. Participants started the next trial when ready. The six buttons illuminated three times each in a random order, for a total of 18 trials. The experimenter recorded the hand (left or right) used to respond to the illuminated button on each trial. For the data analyses, these responses were recoded to represent reach responses executed with the dominant hand. Participants completed the free-reach task in Session 1, prior to engaging in the action-planning task.

\section{Stimuli, responses, and procedure for the action-planning} task

Event A Event A ( $3.5^{\circ}$ of visual angle) was a white arrowhead pointing to the left $(<)$ or right $(>)$ with an asterisk above or below it, or two white arrowheads pointing in opposite directions $(<>)$. Event A appeared on a CRT with the arrowhead(s) centered $1.5^{\circ}$ of visual angle above a white central fixation cross on a black background. The action for Event A (Action A) required a sequence of keypresses with the left or right hand. The arrowhead direction (left or right) indicated the response hand (left or right, respectively), and the location of the asterisk indicated the movement direction of the index finger relative to the center key on the keypad. An asterisk above the arrowhead indicated an "up" response (press the key above the center key, toward the CRT), and an asterisk below the arrowhead indicated a "down" response (press the key below the center key, toward the participant's body). All key responses ("up" or "down") began and ended by pressing the center key. When Event A was a left and a right arrowhead $(<>)$, no response was planned or produced.

Event B Event B was a white light emanating from one of the six circular buttons located on the pedestals covered with black construction paper (see Fig. 1). Only one button, located $15^{\circ}, 35^{\circ}$, or $54^{\circ}$ to the left or right of the participant's body midline, illuminated on each trial. Participants moved one of their hands from the center key on the keypad and pressed the illuminated button with the index finger. The action for Event B (Action B) required a speeded, left-hand reach when a leftside button illuminated and a speeded, right-hand reach when a right-side button illuminated. Thus, reach responses were not executed freely; they were always executed with the limb ipsilateral to the stimulus location, similar to those in Wiediger and Fournier (2008).

\section{Procedure}

Figure 2 shows the trial events. Only two pedestals, at equally eccentric locations (e.g., $15^{\circ}$ left and $15^{\circ}$ right of body midline) were relevant on a given block of trials. At the beginning of each block, the experimenter informed the participants which pair of pedestals (located at $15^{\circ}, 35^{\circ}$, or $54^{\circ}$ ) was relevant for the current block of trials. Participants rested their left and right index fingers on the center keys of the left and right keypads, respectively, before and after responding to the stimulus events. Each trial was initiated by pressing the center 


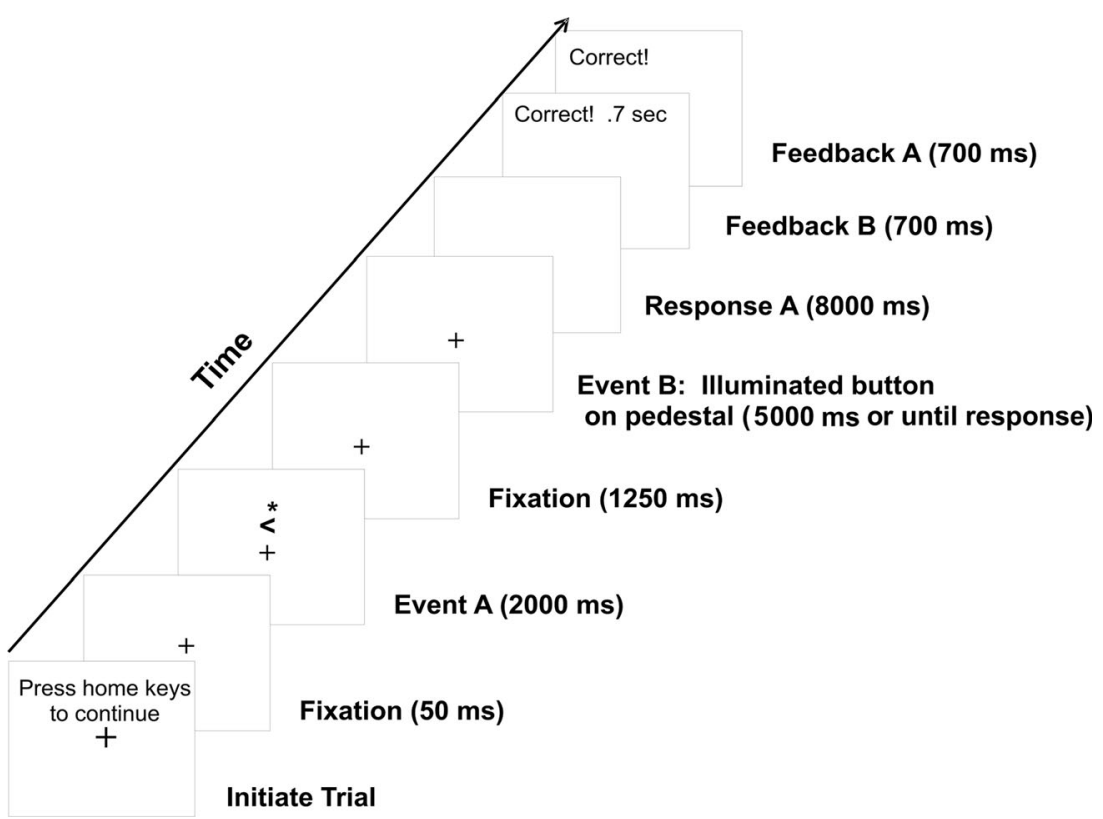

Fig. 2 Trial sequence of events during the action-planning task

(home) keys on the keypads simultaneously. Afterward, the fixation cross appeared at screen center for $50 \mathrm{~ms}$. Next, Event A appeared above the cross for 2,000 ms, followed by the cross alone for 1,250 ms. During this interval, participants planned a response to Event A (Action A), if one was required. Event $B$ then appeared for 5,000 ms or until a response (Action B) was detected. Participants were instructed to execute Action B quickly and accurately. The Action B RT was calculated from the onset of Event $\mathrm{B}$ to the buttonpress response. After executing Action B, participants had 8,000 ms to execute Action A (if required), with an emphasis on accuracy and not speed. After completing the Action A response, the RT and accuracy feedback for Action B and accuracy feedback for Action A appeared sequentially on the screen for $700 \mathrm{~ms}$ each. Then the initiation screen for the next trial appeared. Participants initiated a new trial when ready.

Participants were instructed not to execute any part of Action A until after they had executed Action B. Also, they were not to move fingers or use external cues to help them remember Action A; they were instructed to hold Action A in memory. An experimenter observed participants during the task. If they moved their fingers between the presentations of Event A and Event B during the experimental trials, their data were excluded from the analyses; this occurred for six participants.

\section{Design}

Four factors were varied. First, Handedness varied between participants - participants were either strongly left-hand dominant (i.e., -7 or less on the Edinburgh Handedness Inventory; $3+$ of 4 total left responses on the Lateral Preference
Inventory) or were strongly right-hand dominant (i.e., +7 or greater on the Edinburgh Handedness Inventory; $3+$ of 4 total right responses on the Lateral Preference Inventory. Second, feature overlap between Action B and Action A was manipulated within participants: The hands required to execute Action B and Action A were either the same (feature overlap), different (no overlap), or no Action A was planned (no planning). The difference in the RTs to Action B in the feature overlap and no-overlap conditions served as the measure of partial repetition benefits or costs. Third, the hand used to execute Action B (the reach hand) was either the participant's dominant or nondominant hand (manipulated within participants). Fourth, the Event B eccentricity was varied between blocks (within participants), so that the pair of pedestals (right and left) at which Event B occurred was either at $15^{\circ}, 35^{\circ}$ or $54^{\circ}$ from body midline.

Participants completed the action-planning task over two sessions. Session 1 required one practice block and 12 experimental blocks of 28 trials; Session 2 required one practice block and 15 experimental blocks of 28 trials. The eccentricity of Event B $\left(15^{\circ}, 35^{\circ}\right.$ or $\left.54^{\circ}\right)$ was varied across blocks with equal probabilities of occurrence. Thirty unique block orders representing the three different Event B eccentricities were constructed. The block orders were counterbalanced within and between left- and right-handed participants, such that the same eccentricity was not run in sequence. Each of the 30 block orders was assigned to one left- and one right-handed participant. Within each block of trials, the four different arrowhead and asterisk combinations appeared six times each and the two arrowheads (i.e., $<>$ ), representing the noplanning condition, appeared four times. Event A stimuli were 
paired with the Event B locations (left and right) equally often within each block of trials. At the end of Session 2, a debriefing questionnaire was administered to assess task strategies.

\section{Results}

\section{Free-reach task}

A Handedness (left dominant, right dominant) $\times$ Response Side (ipsilateral or contralateral to the dominant hand) $\times$ Stimulus Eccentricity $\left(15^{\circ}, 35^{\circ}, 54^{\circ}\right)$ mixed-design analysis of variance (ANOVA) was conducted on the frequency of responses executed with the dominant hand. Figure 3 shows that both right- and left-handers preferred to use their dominant hand when the stimulus appeared ipsilateral to the dominant hand and when the stimulus appeared near the body midline contralateral to the dominant hand. We observed main effects of response side $[F(1,58)=225.95, p<$ $\left..0001, \eta_{\mathrm{p}}{ }^{2}=.80\right]$ and stimulus eccentricity $[F(2,116)=$ $\left.52.26, p<.0001, \eta_{\mathrm{p}}{ }^{2}=.47\right]$. There was also a two-way interaction between response side and stimulus eccentricity $\left[F(2,116)=45.88, p<.0001, \eta_{\mathrm{p}}{ }^{2}=.44\right]$, but no other effects from the ANOVA were significant, including the three-way interaction $(F<1)$. Planned comparisons showed that both left- and right-handed participants reached with their dominant hand more frequently to all three stimulus locations ipsilateral $\left(M \mathrm{~s}=94 \%, 96 \%\right.$, and $96 \%$ for the $15^{\circ}$, $35^{\circ}$, and $54^{\circ}$ locations, respectively) versus contralateral $\left(M \mathrm{~s}=62 \%, 26 \%\right.$, and $17 \%$ for the $15^{\circ}, 35^{\circ}$, and $54^{\circ}$ locations, respectively) to the dominant hand $(p s<.001)$. Also, both left- and right-handed participants reached with their dominant hand more frequently to the $15^{\circ}$ location contralateral to the dominant hand $(M=62 \%)$ than to the $35^{\circ}$ and $54^{\circ}$ locations contralateral to the dominant hand $(M \mathrm{~s}=26 \%$ and $17 \%$, respectively; $p \mathrm{~s}<.001)$. These free-reach response patterns were equally strong for left- and righthanded participants $(F<1)$.

This pattern of reach responses is consistent with findings by Stins et al. (2001) and the interpretations by Kim et al. (2007; Kim et al., 2011), indicating that both hand dominance and motor complexity determined the frequency patterns of natural (preferred) reach responses to these different stimulus locations. Because there is a stronger tendency to reach with the dominant hand to near locations $\left(15^{\circ}\right)$ than to those farther away $\left(35^{\circ}\right.$ and $\left.54^{\circ}\right)$, there should be more competition between the hands when reaching with the nondominant hand to the near locations in the actionplanning task. As a result, spatially compatible reaches to the near stimulus locations may require more cognitive control (particularly with the nondominant hand), whereas spatially compatible reaches to the more peripheral stimulus locations may utilize more automatic control.

\section{Action-planning task}

A Handedness (left dominant, right dominant $) \times$ Reach Hand (dominant hand, nondominant hand) $\times$ Feature Overlap (overlap, no overlap, no plan) $\times$ Event B Eccentricity $\left(15^{\circ}, 35^{\circ}\right.$, $54^{\circ}$ ) mixed-design ANOVA was conducted separately on the mean correct RTs for Action B and the percent errors for Action A. ${ }^{1}$ The RT analysis for Action B was restricted to trials in which Action $\mathrm{B}$ and Action A were correct. Additionally, Action B RTs more than four standard deviations from the mean for each condition within each participant were removed $(0.6 \%$ of the total trials). Post hoc comparisons were conducted using the Tukey HSD test $(p<.05)$.

Action A percent error The average error rate for Action A was $4.14 \%$. This low error rate suggests that participants did not have difficulty recalling the planned Action A response. Table 1 shows the error rates for each reach hand, Event B eccentricity, and feature overlap condition. A two-way interaction emerged between feature overlap and Event B eccentricity $\left[F(2,104)=3.54, p<.05, \eta_{\mathrm{p}}{ }^{2}=.11\right]$, and a three-way interaction among reach hand, feature overlap, and Event $\mathrm{B}$ eccentricity $\left[F(2,104)=4.36, p<.05, \eta_{\mathrm{p}}^{2}=.08\right]$. Because the sizes of these effects were small $(\sim 1 \%)$, are difficult to interpret, and are not directly relevant to our hypothesis, they are not discussed further. No other effects were significant. Importantly, the small error rates across the different conditions of reach hand, Event B eccentricity, and feature overlap indicate that the number of Action B trials excluded (due to Action A errors) was small. As a result, our interpretations of Action B performance across conditions should not be compromised.

Action B reach RT As is evident in Fig. 4, left-handed and right-handed participants showed the same general pattern of reach RTs with their dominant and nondominant hands for the different feature overlap conditions at the different Event B eccentricities. In particular, reach RTs for the feature overlap and no-overlap conditions were not different at the Event B eccentricities closest to body midline $\left(15^{\circ}\right)$, but RTs for the feature overlap condition were shorter than those for the nooverlap conditions at the Event B eccentricities farthest from body midline $\left(54^{\circ}\right)$ - indicating a partial repetition benefit.

\footnotetext{
${ }^{1}$ There were only four total trials on which a person responded incorrectly to Event B, and these trials were removed from the mean correct Action B RT analysis. Also, the Action A RT was not evaluated, since the Action A RT is confounded by whether Action B required the same hand as Action A or the different hand. Participants were instructed to focus on accurately recalling Action A and not to focus on speed in recalling Action A. We were interested in how responses to Action B would be affected by accurately maintaining the plan for Action A in WM.
} 


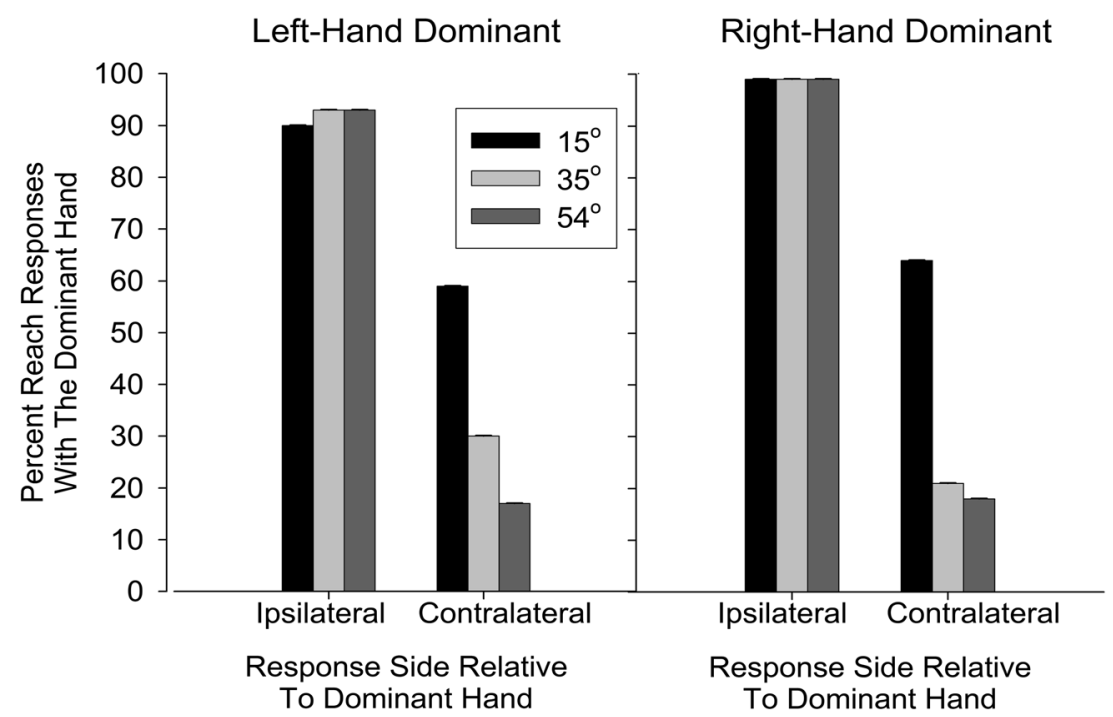

Fig. 3 Free-reach task: Percentages of reach responses with the dominant hand for left-hand-dominant (left panel) and right-hand-dominant (right panel) participants to locations $15^{\circ}, 35^{\circ}$, and $54^{\circ}$ from body midline either

ipsilateral or contralateral to the dominant hand. Error bars show one standard error of the mean

This pattern is not what would be expected if partial repetition benefits were influenced by the hemisphere controlling the hand or the reach-hand preference (from the free-reach data). Significant main effects of feature overlap $[F(2,104)=62.93$, $\left.p<.0001, \eta_{\mathrm{p}}{ }^{2}=.55\right]$ and Event B eccentricity $[F(2,104)=$ $\left.12.84, p<.0001, \eta_{\mathrm{p}}{ }^{2}=.20\right]$ were apparent. In addition, there was a significant two-way interaction between feature overlap and Event B eccentricity $\left[F(4,208)=9.40, p<.0001, \eta_{\mathrm{p}}{ }^{2}=\right.$ .15] and significant three-way interactions among handedness, feature overlap, and Event B eccentricity $[F(4,208)=$ $\left.3.29, p<.02, \eta_{\mathrm{p}}{ }^{2}=.06\right]$ and among handedness, reach hand, and Event B eccentricity $\left[F(2,104)=18.60, p<.0001, \eta_{\mathrm{p}}{ }^{2}=\right.$ .26]. These results show that the reach hand (dominant or nondominant) did not have an influence on feature overlap. Reach hand only influenced the speed of responding to the different Event B eccentricities, which also differed depending on handedness (and is described below). Because our main focus concerned how feature overlap influences reach responses, the three-way interaction involving handedness, feature overlap, and Event B eccentricity is plotted in Fig. 5. Both three-way interactions involving the between-subjects factor Handedness were initially evaluated in separate, repeated measures ANOVAs for left-handed and right-handed participants.

\section{Feature overlap by Event B eccentricity}

For left-handed and right-handed participants, the Feature Overlap $\times$ Event B Eccentricity ANOVA showed a significant effect of feature overlap $\left[F(2,56)=29.06, p<.0001, \eta_{\mathrm{p}}{ }^{2}=\right.$ .51 , and $F(2,48)=34.31, p<.0001, \eta_{\mathrm{p}}{ }^{2}=.59$, respectively], a significant effect for Event B eccentricity $[F(2,56)=7.13, p<$ $.005, \eta_{\mathrm{p}}{ }^{2}=.20$, and $F(2,48)=8.19, p<.001, \eta_{\mathrm{p}}{ }^{2}=.26$, respectively], and a significant interaction between these two factors $\left[F(4,112)=9.67, p<.0001, \eta_{\mathrm{p}}{ }^{2}=.26\right.$, and $F(4,96)=$ $2.69, p<.04, \eta_{\mathrm{p}}{ }^{2}=.10$, respectively] for reach RTs. For lefthanded participants, post hoc comparisons showed that reach RTs were shorter in the feature overlap than in the no-overlap trials at both the $35^{\circ}$ and $54^{\circ}$ Event B eccentricities $(p \mathrm{~s}<.04)$, indicating a partial repetition benefit; but reach RTs did not differ between the feature overlap and no-overlap trials at the $15^{\circ}$ eccentricity $(p>.77)$, indicating that no partial repetition benefit or cost was found at this location. See the left panel in Fig. 5. A different pattern was found for right-handed

Table 1 Action A percent errors (one standard error of the mean) corresponding to reach hand (dominant, nondominant), Event B eccentricity ( $15^{\circ}$, $35^{\circ} 54^{\circ}$ ), and feature overlap (overlap, no overlap)

\begin{tabular}{|c|c|c|c|c|c|c|}
\hline \multirow[t]{3}{*}{ Feature Overlap } & \multicolumn{6}{|c|}{ Reach Hand } \\
\hline & \multicolumn{3}{|l|}{ Dominant } & \multicolumn{3}{|c|}{ Nondominant } \\
\hline & $15^{\circ}$ & $35^{\circ}$ & $54^{\circ}$ & $15^{\circ}$ & $35^{\circ}$ & $54^{\circ}$ \\
\hline Overlap & $3.1 \%(0.4)$ & $4.1 \%(0.6)$ & $4.5 \%(0.5)$ & $4.4 \%(0.7)$ & $3.6 \%(0.7)$ & $4.7 \%(0.7)$ \\
\hline No overlap & $4.2 \%(0.5)$ & $3.9 \%(0.5)$ & $4.5 \%(0.6)$ & $4.4 \%(0.6)$ & $4.6 \%(0.7)$ & $3.5 \%(0.5)$ \\
\hline
\end{tabular}




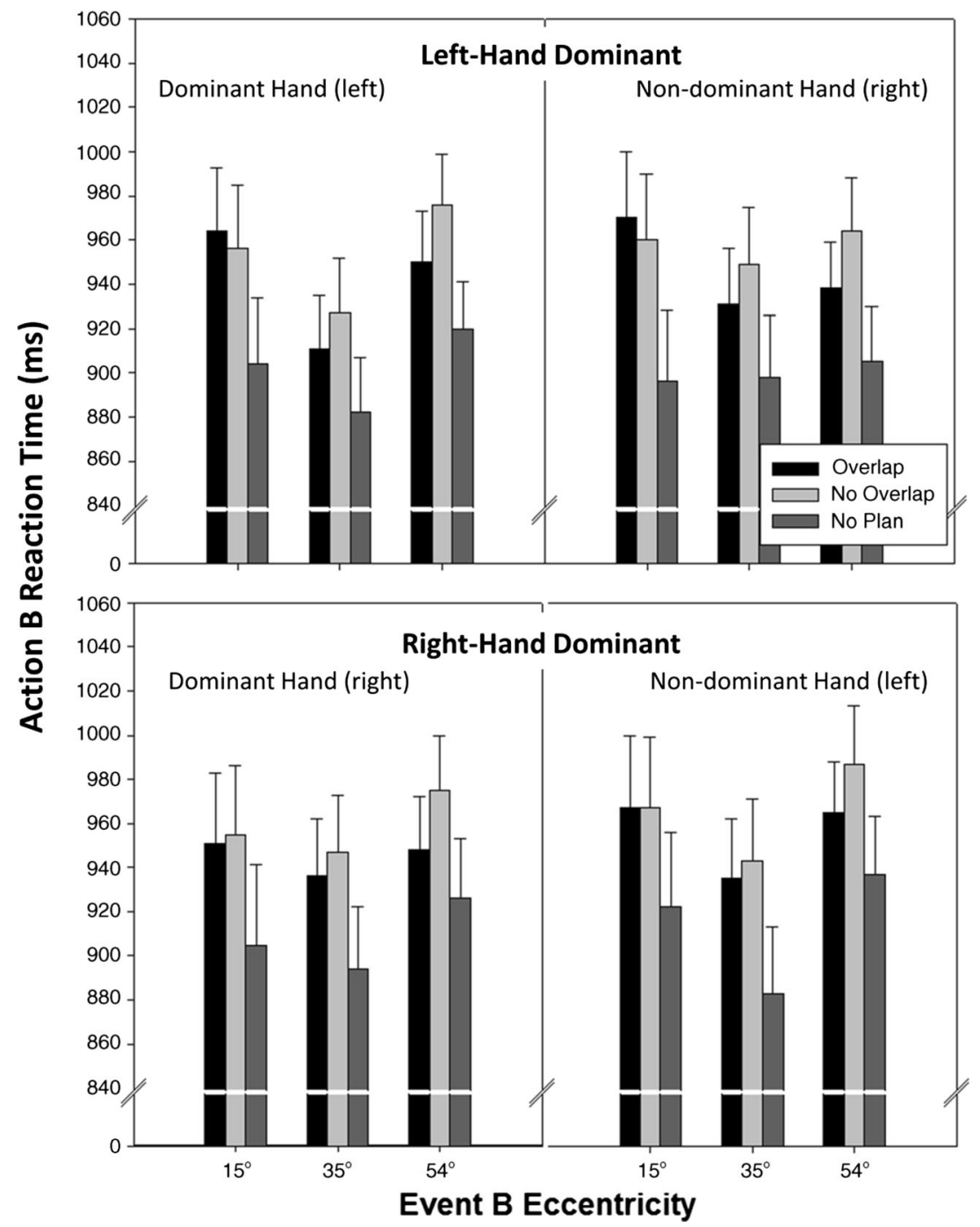

Fig. 4 Action-planning task: Action B correct reaction times for lefthand-dominant (upper panels) and right-hand-dominant (lower panels) participants for each reach hand (dominant hand = left panels; nondominant hand $=$ right panels), Event $\mathrm{B}$ eccentricity $\left(15^{\circ}, 35^{\circ}\right.$, and $54^{\circ}$ ), and feature overlap (overlap, no overlap, and no plan). Error bars show one standard error of the mean participants. For these participants, post hoc comparisons showed that reach RTs were shorter in the feature overlap than in the no-overlap trials at the $54^{\circ}$ eccentricity $(p<.001)$, indicating a partial repetition benefit; but reach RTs did not differ between the feature overlap and no-overlap trials at the $15^{\circ}$ and $35^{\circ}$ Event $\mathrm{B}$ eccentricities ( $p \mathrm{~s}>.82$ ), indicating that no partial repetition benefit or cost was found at these locations. See the right panel in Fig. 5. Thus, both left- and right-handed participants showed no partial repetition benefit or cost at the $15^{\circ}$ Event B eccentricity, and both left- and right-handed participants showed a partial repetition benefit at the $54^{\circ}$ eccentricity. Additionally, left-handed participants showed a partial repetition benefit at the shorter reach eccentricity of $35^{\circ}$.

Furthermore, post hoc comparisons showed that the reach RTs in both the feature overlap and no-overlap trials were longer than those in the no-planning trials at all three Event B eccentricities $(p \mathrm{~s}<.001)$. This finding indicates that the participants were maintaining Action A in short-term memory or WM during the action-planning trials while executing their reach responses (e.g., Jolicœur \& Dell'Acqua, 1998; Logan, 1979). Implications of this finding are addressed in the Discussion.

To test whether the partial repetition benefit (reported above) for left-handed participants occurred at a shorter reach eccentricity $\left(35^{\circ}\right)$ than that for right-handed participants $\left(54^{\circ}\right)$, a Handedness $\times$ Event B Eccentricity mixed-design ANOVA was conducted on RT differences between the no-featureoverlap and overlap conditions (partial repetition benefit). The results showed a significant main effect of Event B eccentricity $\left[F(2,104)=26.85, p<.001, \eta_{\mathrm{p}}{ }^{2}=.34\right]$ but did not show a significant main effect of handedness $(F<1)$ or an interaction between handedness and Event $\mathrm{B}$ eccentricity $\left[F(2,104)=2.95, p=.06, \eta_{\mathrm{p}}{ }^{2}=.05\right]$. The lack of a significant interaction suggests that RTs reflecting partial repetition benefits did not vary differently across the Event B eccentricities 


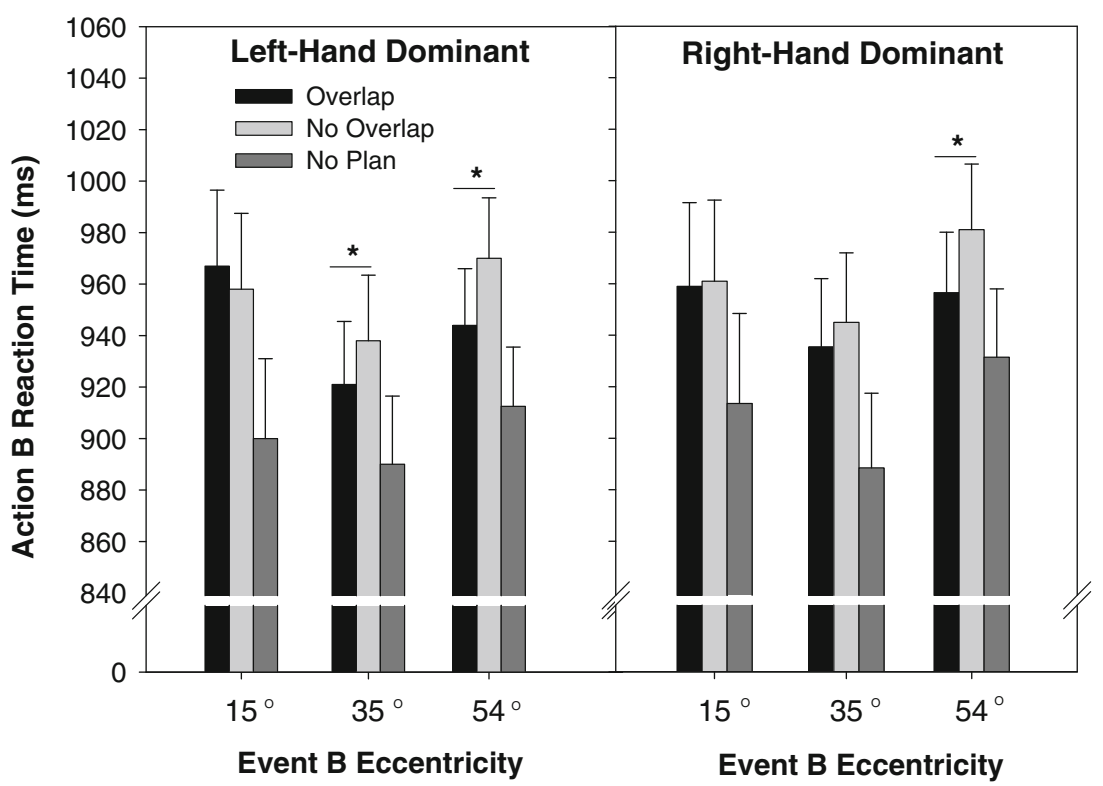

Fig. 5 Action-planning task: Action B correct reaction times (RTs) for left-hand-dominant (left panel) and right-hand-dominant (right panel) participants for the feature overlap conditions (overlap, no overlap, and no plan) at each Event B eccentricity $\left(15^{\circ}, 35^{\circ}\right.$, and $54^{\circ}$ ), collapsed across reach hands (dominant, nondominant). Error bars show one standard error of the mean. No partial repetition cost or benefit was found at the $15^{\circ}$ Event B eccentricity. Partial repetition benefits were significant $(*)$ for

left-hand-dominant participants at the $35^{\circ}$ and $54^{\circ}$ Event B eccentricities, and for right-hand-dominant participants at the $54^{\circ}$ Event B eccentricity; but the benefit at $35^{\circ}$ for the left-hand dominant participants was not statistically greater than that found at $35^{\circ}$ for the right-hand-dominant participants. Also, RTs for both the overlap and no-overlap conditions were longer than those in the no-plan condition at all Event B eccentricities, indicating a dual-task decrement

for left- and right-handers. This was confirmed by contrasts that compared the benefits found for left- and right-handers at the $15^{\circ}(p=.28), 35^{\circ}(p=.24)$, and $54^{\circ}(p=.81)$ eccentricities. Also, the main effect found for Event B eccentricity shows that the RT difference between the feature overlap and nooverlap conditions increased with increases in Event B eccentricity. Post hoc analyses showed that the RT difference between the no-feature-overlap and overlap conditions was greatest at the $54^{\circ}$ eccentricity, intermediate at the $35^{\circ}$ eccentricity, and smallest at the $15^{\circ}$ eccentricity $(p \mathrm{~s}<.05)$. Taken together, we can conclude that both left- and right-handed participants showed a partial repetition benefit at the farthest reach eccentricity $\left(54^{\circ}\right)$ and showed no partial repetition benefit or cost at the closest reach eccentricity $\left(15^{\circ}\right)$. See Fig. 5 .

\section{Reach hand and Event $B$ eccentricity}

For both left-handed and right-handed participants, the Reach Hand $\times$ Event B Eccentricity ANOVA showed a significant effect of Event B eccentricity $[F(2,56)=13.09, p<.0001$, $\eta_{\mathrm{p}}{ }^{2}=.32$, and $F(2,48)=6.94, p<.01, \eta_{\mathrm{p}}{ }^{2}=.22$, respectively] and a significant interaction $\left[F(2,56)=15.10, p<.0001, \eta_{\mathrm{p}}{ }^{2}=\right.$ .35 , and $F(2,48)=4.41, p<.02, \eta_{\mathrm{p}}{ }^{2}=.16$, respectively] for reach RTs. For left-handed participants, reach responses were equally fast with the dominant and nondominant hands to the $15^{\circ}$ Event B eccentricities $(p>.68)$ and were faster with the dominant than with the nondominant hand to the $35^{\circ}$ Event $\mathrm{B}$ eccentricities $(p<.005)$, with a reverse trend at the $54^{\circ}$

eccentricities $(p=.053)$. Right-handed participants showed a different pattern: Reach responses were faster with the dominant than with the nondominant hand to the $15^{\circ}$ Event $\mathrm{B}$ eccentricities $(p<.05)$, were equally fast with both hands at the $35^{\circ}$ eccentricities $(p=1)$, and were faster with the dominant hand to the $54^{\circ}$ eccentricities $(p<.04)$. We do not have an explanation for this pattern of responses based on handedness. Importantly, this qualitative difference does not compromise the interpretation of our results in terms of the hypotheses being investigated.

\section{Discussion}

In this study, we examined whether partial repetition benefits occur for reach responses to a spatially compatible stimulus assumed to evoke automatic control (Stürmer, et al., 2002; Tagliabue et al., 2000; Umiltà \& Nicoletti, 1990). This study also examined whether these benefits are influenced by the hemisphere controlling the reaching hand or by the reach-hand preference (defined by the free-reach task) in left- and right-handed individuals. Left- and right-handed individuals showed neither a partial repetition benefit nor a cost for spatially compatible reaches closest to body midline $\left(15^{\circ}\right)$; reach RTs did not differ between the feature overlap and no-overlap conditions. However, left- and right-handed individuals showed a partial repetition benefit for the spatially compatible reaches farthest from body midline $\left(54^{\circ}\right)$; reach RTs were shorter for the feature overlap than 
for the no-overlap condition. Thus, partial repetition benefits were contingent on the angular distance of the spatially compatible reach responses from body midline. These benefits were not influenced by the hemisphere controlling the reach hand (left or right) or by reach-hand preference. Furthermore, no partial repetition costs were observed at any of the reach locations. Provided that the spatially compatible reach responses in the present study were automatically activated, this finding shows that partial repetition costs do not occur for reach responses that are activated automatically (e.g., Fournier et al., 2010; Wiediger \& Fournier, 2008). These findings suggest that automatic reach actions did not reactivate (prime) the action plan in WM with a shared action feature, to create code confusion and selection competition and thus result in reach response delays (a partial repetition cost). Instead, automatic reach actions, at least in some cases, benefited from response priming by the plan in WM, resulting in reach response facilitation (a partial repetition benefit).

The results also showed a robust dual-task decrement for reach responses when concurrently committing and retaining an action plan in WM to be executed later. That is, spatially compatible reach responses to all stimulus locations were slower for the feature overlap and no-overlap conditions than for the no-planning condition. This dual-task decrement does not indicate that activating the specific reach response (left or right hand) required access to WM (i.e., required cognitive control). Instead, this dual-task decrement can be attributed to an executive control mechanism involved in coordinating the execution order of the reach action and the action maintained in WM (e.g., execute the reach action immediately while inhibiting the previously activated action; see Monsell, 2003). Coordinating the execution of two different action plans was only necessary in the feature overlap and no-overlap conditions, not in the noplanning condition (this condition required only a reach action). Importantly, this dual-task decrement suggests that participants maintained the action plan to the first stimulus event in WM while executing the reach response (see, e.g., Behmer \& Fournier, 2014; Jolicœur \& Dell'Acqua, 1998; Logan, 1979).

In contrast to our predictions, partial repetition benefits were not influenced by the hemisphere controlling the hand or by reach-hand preference (Wiediger \& Fournier, 2008). Instead, partial repetition benefits were influenced by the angular distance of the spatially compatible reaches from body midline. If the hemisphere controlling the hand did have an influence, benefits should have occurred for spatially compatible reaches to the right, but not to the left of body midline - assuming that the left hemisphere is specialized for automatic visuo-motor control (e.g., Gonzalez et al., 2006). If reach-hand preference influenced partial repetition benefits, the location of these benefits should have resembled the reach-hand preferences found in the free-reach task. The free-reach task revealed a largely consistent reach-hand preference with the spatially compatible hand (regardless of hand dominance) when the stimulus was far from $\left(54^{\circ}\right)$ but not when it was near $\left(15^{\circ}\right)$ body midline. When the stimulus was near body midline $\left(15^{\circ}\right)$, there was a preference among both left- and right-handed individuals to reach with the dominant hand, even when it was spatially incompatible. As a result, competition between the hands, and hence more cognitive control (e.g., Eimer et al., 1995; Kornblum et al., 1990), for both left- and right-handers was expected in the action-planning task when reaching to near $\left(15^{\circ}\right)$ locations with the nondominant hand. However, we found no evidence that the partial repetition benefits (or costs) in our study were influenced by hand dominance when executing a spatially compatible response to the $15^{\circ}$ locations.

Partial repetition benefits may be accounted for by the degree of feature overlap between the action plan maintained in memory and the current action. Consistent with this idea, research shows that increasing feature overlap between these two action plans when both are under cognitive control can lead to greater partial repetition costs (see Hommel et al., 2001; Stoet \& Hommel, 1999; see also the review by Thomaschke et al., 2012a). Thus, it is possible that increasing the feature overlap between two action plans that are both under automatic control can lead to greater partial repetition benefits. In our study, feature overlap (left or right) was likely strongest for the reach actions most peripheral to body midline $\left(54^{\circ}\right)$. This is because the six buttons that presented a stimulus at the $15^{\circ}, 35^{\circ}$, and $54^{\circ}$ locations to the left and right of body midline were present during all trials. As a result, a stimulus presented at one of the button locations farthest from body midline may be more strongly representative of a "left" or a "right" stimulus than is a stimulus presented at one of the button locations closest to body midline. We confirmed this by asking 15 volunteers (graduate students and faculty in our department who had no knowledge about our study) to sit in front of our apparatus (with their hands resting on the response keys on the table) and to reach to a left button and then to a right button. The results showed that 13 of them executed spatially compatible reach responses to the buttons at the $54^{\circ}$ locations, two of them executed spatially compatible reach responses to the buttons at the $34^{\circ}$ locations, and none of them reached to the buttons at the $15^{\circ}$ locations. Thus, the action maintained in WM requiring a left or a right response may have more effectively primed the compatible features ("left" or "right") for a stimulus appearing at the $54^{\circ}$ location (leading to a partial repetition benefit) because the features at this location were more strongly represented as "left" and "right" than those at the $15^{\circ}$ location. ${ }^{2}$

\footnotetext{
${ }^{2}$ We also examined whether the hand movement direction for Action A (up = toward the CRT, down = toward body) in addition to reach side (left, right) had an effect on reach responses at the different stimulus-reach eccentricities. That is, it was possible that planned up and down movements on the keypad for Action A overlapped more and less, respectively, with reach responses to the $15^{\circ}$ location (which required a forward reach) than with the other reach locations. We found no evidence, however, to support this possibility. See the supplemental information for an analysis.
} 
The multiple stimulus-reach locations in the present study may also account for why our results differed from those obtained by Wiediger and Fournier (2008) at comparable stimulus-reach locations. Because a variety of stimulus-reach eccentricities were arranged to the left and the right in the present study $\left(15^{\circ}, 35^{\circ}\right.$, and $\left.54^{\circ}\right)$, but only one in the Wiediger and Fournier study $\left(17^{\circ}\right)$, the participants' representations of left and right at the $15^{\circ}$ locations (present study) and $17^{\circ}$ locations (Wiediger \& Fournier, 2008) were likely different. That is, the stimulus locations in the Wiediger and Fournier study may have been defined more clearly and represented more strongly as "left" or "right," because these were the only locations available to define "left" and "right." As a result, these codes should be more strongly activated to be primed by and/or to compete with the action codes retained in WM at the close stimulusreach locations in their study than in ours. Recall that they found a significant partial repetition benefit for spatially compatible reach responses with the dominant (right) hand and a significant partial repetition cost for spatially compatible reach responses with the nondominant (left) hand. This pattern is consistent with what we would expect, given our free-reach pattern of responses reflecting reach-hand preference: facilitation when reaching with the spatially compatible preferred hand, and competition when reaching with the spatially compatible nonpreferred hand. Thus, it is possible that reach-hand preference contributes to the degree of automatic control involved in reaching to a spatially compatible stimulus, as was suggested by Wiediger and Fournier. However, reaching with the preferred versus the nonpreferred hand may not reveal significant partial repetition benefits if the reach responses are only weakly primed by the action retained in WM.

Although we argue that the partial repetition benefits found in the present study were due to the action plan maintained in WM priming the reach response under automatic control, automatic shifts in attention resulting from the action plan maintained in WM may have contributed to the partial repetition benefits found (see the review by Thomaschke et al., 2012a). If maintaining a left- or right-hand response in WM caused a shift in attention to the area of space (left or right) corresponding to the action hand (see, e.g., Reed, Grubb, \& Steele, 2006), we would expect to find slower reach responses for the nofeature-overlap condition (attention cost) than for the feature overlap and no-planning conditions at the farthest stimulusreach eccentricity $\left(54^{\circ}\right)$. This, in fact, was what we observed. Moreover, we should find a larger difference in reach RTs between the no-feature-overlap and no-planning conditions at the farthest stimulus-reach eccentricity $\left(54^{\circ}\right)$ relative to the closest ( $15^{\circ}$; attention shift cost). However, this was not observed. (See Fig. 5.) We conducted a Handedness (left, right) $\times$ Feature Overlap (overlap, no overlap) $\times$ Reach Hand (dominant, nondominant $) \times$ Event B Eccentricity $\left(15^{\circ}, 35^{\circ}, 54^{\circ}\right)$ mixed-design ANOVA on the reach RT differences between the no-planning and feature overlap conditions. The results showed a significant main effect of feature overlap $[F(1,52)$ $\left.=13.27, p<.001, \eta_{\mathrm{p}}^{2}=.203\right]$ and a significant two-way interaction between feature overlap and Event B eccentricity $\left[F(2,104)=22.86, p<.001, \eta_{\mathrm{p}}{ }^{2}=.341\right]$. Post hoc analyses (Tukey HSD) showed that the difference in RTs between the overlap and no-planning trials was smallest at the $54^{\circ}$, intermediate at the $35^{\circ}$, and largest at the $15^{\circ}(p \mathrm{~s}<.001)$ stimulusreach eccentricities. Importantly, however, the differences in RTs between the no-overlap and no-planning trials (attention shift cost) did not differ across the three stimulus-reach eccentricities $(p s>98)$. Thus, shifts in attention by the action plan maintained in memory cannot account for the partial repetition benefits found at the more peripheral stimulus locations. Also, shifts in attention cannot account for the partial repetition costs and benefits found by Wiediger and Fournier (2008).

In summary, we showed that reach actions under automatic control are not hindered, but can be facilitated (primed) by an action plan retained in WM when the retained action shares a feature with the current action (partial repetition benefit). Previous research had shown that actions under cognitive control were hindered by an action plan retained in WM when the retained action shared a feature with the current action (partial repetition cost). Our findings are consistent with research demonstrating that actions carried out automatically (with little or no cognitive control) are susceptible to facilitative priming (Brass, Bekkering, Wohlschläger, \& Prinz, 2000; Craighero, Bello, Fadiga, \& Rizzolatti, 2002; Craighero, Fadiga, Rizzolatti, \& Umiltà, 1998, 1999). Our findings, together with previous research, indicate that partial repetition benefits and costs depend on whether the current action is under more automatic or under more cognitive control (Fournier, Behmer, \& Stubblefield, 2014a) and on the degree of feature overlap between the current action and the action plan retained in WM (Hommel et al., 2001; Proctor et al., 1995; Stoet \& Hommel, 1999; Thomaschke et al., 2012a). A partial repetition cost will occur when the current action requires WM and reactivates the other plan maintained in WM that has similar features, leading to code confusion and selection competition. If the current action is automatic, it will not reactivate another plan maintained in WM that has similar features, and hence will not lead to code confusion and selection competition. Instead, the current action may benefit from priming by the action plan maintained in $\mathrm{WM}$, leading to a partial repetition benefit.

\section{References}

Anderson, J. R. (1992). Automaticity and the ACT* theory. American Journal of Psychology, 105, 165-180.

Barber, P., \& O'Leary, M. (1997). The relevance of salience: Towards an activational account of irrelevant stimulus-response compatibility 
effects. In B. Hommel \& W. Prinz (Eds.), Theoretical issues in stimulus-response compatibility (pp. 135-172). Amsterdam: North-Holland.

Behmer, L. P., Jr., \& Fournier, L. R. (2014). Working memory modulates neural efficiency over motor components during a novel action planning task: An EEG study. Behavioural Brain Research, 260, 1-7. doi:10.1016/j.bbr.2013.11.031

Brass, M., Bekkering, H., Wohlschläger, A., \& Prinz, W. (2000). Compatibility between observed and executed finger movements: Comparing symbolic, spatial, and imitative cues. Brain and Cognition, 44, 124-143. doi:10.1006/brcg.2000.1225

Cohen, J. D., Dunbar, K., \& McClelland, J. L. (1990). On the control of automatic processes: A parallel distributed processing account of the Stroop effect. Psychological Review, 97, 332-361. doi:10.1037/ 0033-295X.97.3.332

Coren, S. (1993). The lateral preference inventory for measurement of handedness, footedness, eyedness, and earedness: Norms for young adults. Bulletin of the Psychonomic Society, 31, 1-3.

Craighero, L., Fadiga, L., Rizzolatti, G., \& Umiltà, C. (1998). Visuomotor priming. Visual Cognition, 5, 109-125. doi:10.1080/713756780

Craighero, L., Fadiga, L., Rizzolatti, G., \& Umiltà, C. (1999). Action for perception: A motor-visual attentional effect. Journal of Experimental Psychology: Human Perception and Performance, 25, 1673-1692. doi:10.1037/0096-1523.25.6.1673

Craighero, L., Bello, A., Fadiga, L., \& Rizzolatti, G. (2002). Hand action preparation influences the responses to hand pictures. Neuropsychologia, 40, 492-502. doi:10.1016/S0028-3932(01) 00134-8

de Jong, R., Liang, C.-C., \& Lauber, E. (1994). Conditional and unconditional automaticity: A dual-process model of effects of spatial stimulus-response correspondence. Journal of Experimental Psychology: Human Perception and Performance, 20, 731-750. doi:10.1037/0096-1523.20.4.731

Eimer, M. (1995). Stimulus-response compatibility and automatic response activation: Evidence from psychophysiological studies. Journal of Experimental Psychology: Human Perception and Performance, 21, 837-854. doi:10.1037/0096-1523.21.4.837

Eimer, M., Hommel, B., \& Prinz, W. (1995). S-R compatibility and response selection. Acta Psychologica, 90, 301-313. doi:10.1016/ 0001-6918(95)00022-m

Elliot, D., \& Chua, R. (1996). Manual asymmetries in goal-directed movements. In D. Elliot \& E. Roy (Eds.), Manual asymmetries in motor performance (pp. 143-157). New York: CRC Press.

Fisk, J. D., \& Goodale, M. A. (1988). The effects of unilateral brain damage on visually guided reaching: Hemispheric differences in the nature of the deficit. Experimental Brain Research, 72, 425-435.

Fitts, P. M., \& Seeger, C. M. (1953). S-R compatibility: Spatial characteristics of stimulus and response codes. Journal of Experimental Psychology, 46, 199-210. doi:10.1037/h0062827

Fournier, L. R., \& Gallimore, J. M. (2013). What makes an event: Temporal integration of stimuli or actions? Attention, Perception, \& Psychophysics, 75, 1293-1305. doi:10.3758/s13414-013-0461-x

Fournier, L. R., Wiediger, M. D., McMeans, R., Mattson, P. S., Kirkwood, J., \& Herzog, T. (2010). Holding a manual response sequence in memory can disrupt vocal responses that share semantic features with the manual response. Psychological Research, 74, 359-369. doi:10.1007/s00426-009-0256-9

Fournier, L. R., Behmer, L. P., Jr., \& Stubblefield, A. M. (2014a). Interference due to shared features between action plans is influenced by working memory span. Psychonomic Bulletin \& Review, 6, 1524-1529. doi:10.3758/s13423-014-0627-0

Fournier, L. R., Gallimore, J. M., Feiszli, K., \& Logan, G. D. (2014b). On the importance of being first: Serial order effects in the interaction between action plans and ongoing actions. Psychonomic Bulletin \& Review, 21, 163-169. doi:10.3758/s13423-013-0486-0
Gabbard, C., \& Rabb, C. (2001). Imagined and actual limb selection: A test of preference. Brain and Cognition, 46, 139-144.

Gonzalez, C. L. R., Ganel, T., \& Goodale, M. A. (2006). Hemispheric specialization for the visual control of action is independent of handedness. Journal of Neurophysiology, 95, 3496-3501. doi:10.1152/ jn.01187.2005

Greenwald, A. G. (2003). On doing two things at once: III. Confirmation of perfect timesharing when simultaneous tasks are ideomotor compatible. Journal of Experimental Psychology: Human Perception and Performance, 29, 859-868. doi:10.1037/ 0096-1523.29.5.859

Greenwald, A. G., \& Shulman, H. G. (1973). On doing two things at once: II. Elimination of the psychological refractory period effect. Journal of Experimental Psychology, 101, 70-76. doi:10.1037/ h0035451

Hallet, P. E. (1978). Primary and secondary saccades to goals defined by instructions. Vision Research, 18, 1279-1296.

Helbig, C., \& Gabbard, C. (2004). What determines limb selection for reaching? Research Quarterly for Exercise and Sport, 75, $47-59$.

Hommel, B. (2004). Event files: Feature binding in and across perception and action. Trends in Cognitive Science, 8, 494-500. doi:10.1016/j. tics.2004.08.007

Hommel, B. (2005). How much attention does an event file need? Journal of Experimental Psychology: Human Perception and Performance, 31, 1067-1082. doi:10.1037/0096-1523.31.5.1067

Hommel, B., Müsseler, J., Aschersleben, G., \& Prinz, W. (2001). The theory of event coding (TEC): A framework for perception and action planning. Behavioral and Brain Sciences, 24, 849-878. doi: 10.1017/S0140525X01000103

Jolicœur, P., \& Dell'Acqua, R. (1998). The demonstration of short-term consolidation. Cognitive Psychology, 36, 138-202. doi:10.1006/ cogp.1998.0684

Keele, S. W. (1968). Movement control in skilled motor performance. Psychological Bulletin, 70, 387-403. doi:10.1037/h0026739

Kim, W., Gabbard, C., Ryu, Y., \& Buchanan, J. (2007). Right-handers' reaching in contralateral hemispace: A kinematic observation. Journal of Motor Behavior, 39, 451-456.

Kim, W., Buchanan, J., \& Gabbard, C. (2011). Constraints on arm selection processes when reaching: Degrees of freedom and joint amplitudes interact to influence limb selection. Journal of Motor Behavior, 43, 403-411.

Kornblum, S., \& Lee, J.-W. (1995). Stimulus-response compatibility with relevant and irrelevant stimulus dimensions that do and do not overlap with the response. Journal of Experimental Psychology: Human Perception and Performance, 21, 855-875. doi:10.1037/0096-1523.21.4.855

Kornblum, S., Hasbroucq, T., \& Osman, A. (1990). Dimensional overlap: Cognitive basis for stimulus-response compatibility-A model and taxonomy. Psychological Review, 97, 253-270. doi:10.1037/0033295X.97.2.253

Lashley, K. S. (1951). The problem of serial order in behavior. In L. A. Jeffress (Ed.), Cerebral mechanisms in behavior (pp. 112-146). New York: Wiley.

Logan, G. D. (1979). On the use of a concurrent memory load to measure attention and automaticity. Journal of Experimental Psychology: Human Perception and Performance, 5, 189-207. doi:10.1037/ 0096-1523.5.2.189

Logan, G. D. (1988). Toward an instance theory of automatization. Psychological Review, 95, 492-527. doi:10.1037/0033-295X.95.4. 492

Logan, G. D., \& Crump, M. J. C. (2011). Hierarchical control of cognitive processes: The case for skilled typewriting. In B. H. Ross (Ed.), The psychology of learning and motivation (Vol. 54, pp. 1-27). Burlington: Academic Press. 
MacLeod, C. M., \& Dunbar, K. (1988). Training and Stroop-like interference: Evidence for a continuum of automaticity. Journal of Experimental Psychology: Learning, Memory, and Cognition, 14, 126-135. doi:10.1037/0278-7393.14.1.126

Mattson, P. S., \& Fournier, L. R. (2008). An action sequence held in memory can interfere with response selection of a target, but does not interfere with response activation of noise stimuli. Memory \& Cognition, 36, 1236-1247. doi:10.3758/MC.36.7.1236

Mattson, P. S., Fournier, L. R., \& Behmer, L. P., Jr. (2012). Frequency of the first feature in action sequences influences feature binding. Attention, Perception, \& Psychophysics, 74, 1446-1460. doi:10. 3758/s13414-012-0335-7

Meyer, D. E., \& Gordon, P. C. (1985). Speech production: Motor programming of phonetic features. Journal of Memory and Language, 24, 3-26.

Miller, G. A., Galanter, E., \& Pribram, K. H. (1960). Plans and the structure of behavior. New York: Holt, Rinehart \& Winston.

Monsell, S. (2003). Task switching. Trends in Cognitive Sciences, 7, 134 140. doi:10.1016/S1364-6613(03)00028-7

Newell, A., \& Rosenbloom, P. S. (1981). Mechanisms of skill acquisition and the law of practice. In J. R. Anderson (Ed.), Cognitive skills and their acquisition (pp. 1-55). Hillsdale: Erlbaum.

Oldfield, R. C. (1971). The assessment and analysis of handedness: The Edinburgh Inventory. Neuropsychologia, 9, 97-113. doi:10.1016/ 0028-3932(71)90067-4

Passingham, R. E., \& Toni, I. (2001). Contrasting the dorsal and ventral visual systems: Guidance of movement versus decision making. Neurolmage, 14, S125-S131.

Proctor, R. W., Lu, C.-H., Wang, H., \& Dutta, A. (1995). Activation of response codes by relevant and irrelevant stimulus information. Acta Psychologica, 90, 275-286. doi:10.1016/0001-6918(95)00030-X

Radoeva, P., Cohen, J., Corballis, P., Lukovits, T., \& Koleva, S. (2005). Hemispheric asymmetry in a dissociation between the visuomotor and visuoperceptual streams. Neuropsychologia, 43, 1763-1773. doi:10.1016/j.neuropsychologia.2005.02.005

Reed, C. L., Grubb, J. D., \& Steele, C. (2006). Hands up: Attentional prioritization of space near the hand. Journal of Experimental Psychology: Human Perception and Performance, 32, 166-177. doi:10.1037/0096-1523.32.1.166

Rosenbaum, D. A., Chapman, K. M., Weigelt, M., Weiss, D. J., \& van der Wel, R. (2012). Cognition, action, and object manipulation. Psychological Bulletin, 138, 924-946. doi:10.1037/a0027839

Sevald, C. A., \& Dell, G. S. (1994). The sequential cuing effect in speech production. Cognition, 53, 91-127.

Simon, J. R. (1990). The effects of an irrelevant directional cue on human information processing. In R. W. Proctor \& T. G. Reeve (Eds.), Stimulus-response compatibility: An integrated perspective (pp. 31-86). Amsterdam: North-Holland.
Stins, J., Kadar, E., \& Costall, A. (2001). A kinematic analysis of hand selection in a reaching task. Laterality, 6, 347-367.

Stoet, G., \& Hommel, B. (1999). Action planning and the temporal binding of response codes. Journal of Experimental Psychology: Human Perception and Performance, 25, 1625-1640. doi:10.1037/00961523.25.6.1625

Stürmer, B., Leuthold, H., Soetens, E., Schröter, H., \& Sommer, W. (2002). Control over location-based response activation in the Simon task: Behavioral and electrophysiological evidence. Journal of Experimental Psychology: Human Perception and Performance, 28, 1345-1363. doi:10.1037/0096-1523.28.6.1345

Tagliabue, M., Zorzi, M., Umiltà, C., \& Bassignani, F. (2000). The role of long-term-memory and short-term-memory links in the Simon effect. Journal of Experimental Psychology: Human Perception and Performance, 26, 648-670. doi:10.1037/0096-1523.26.2.648

Theeuwes, J., Kramer, A. F., Hahn, S., \& Irwin, D. E. (1998). Our eyes do not always go where we want them to go: Capture of the eyes by new objects. Psychological Science, 9, 379-385. doi:10.1111/14679280.00071

Thomaschke, R., Hopkins, B., \& Miall, R. C. (2012a). The planning and control model (PCM) of motorvisual priming: Reconciling motorvisual impairment and facilitation effects. Psychological Review, 119, 388-407. doi:10.1037/a0027453

Thomaschke, R., Hopkins, B., \& Miall, R. C. (2012b). The role of cueresponse mapping in motorvisual impairment and facilitation: Evidence for different roles of action planning and action control in motorvisual dual-task priming. Journal of Experimental Psychology: Human Perception and Performance, 38, 336-349. doi:10.1037/a0024794

Todor, J., \& Cisneros, J. (1985). Accommodation to increased accuracy demands by the right and left hands. Journal of Motor Behavior, 17, $355-372$.

Umiltà, C., \& Nicoletti, R. (1990). Spatial stimulus-response compatibility. In R. W. Proctor \& T. G. Reeve (Eds.), Stimulus-response compatibility: An integrated perspective (pp. 89-116). Amsterdam: North-Holland.

Umiltà, C., \& Zorzi, M. (1997). Commentary on Barber and O’Leary: Learning and attention in S-R compatibility. In B. Hommel \& W. Prinz (Eds.), Theoretical issues in stimulus-response compatibility (pp. 173-178). Amsterdam: North-Holland.

Wiediger, M., \& Fournier, L. (2008). An action sequence withheld in memory does not necessarily delay execution of a similar action: An investigation of compatibility interference. Journal of Experimental Psychology: Human Perception and Performance, 34, 1136-1149. doi:10.1037/0096-1523.34.5.1136

Yaniv, I., Meyer, D. E., Gordon, P. C., Huff, C. A., \& Sevald, C. A. (1990). Vowel similarity, connectionist models, and syllable structure in motor programming of speech. Journal of Memory and Language, 29, 1-26. 